\title{
Epitaxial CdSe-Au Nanocrystal Heterostructures by Thermal Annealing
}

\author{
Albert Figuerola, ${ }^{\dagger, I l}$ Marijn van Huis, ${ }^{\ddagger}, \S$, II Marco Zanella, ${ }^{\dagger}$ Alessandro Genovese, ${ }^{\dagger}$ \\ Sergio Marras, ${ }^{\dagger}$ Andrea Falqui, ${ }^{\dagger}$ Henny W. Zandbergen, ${ }^{\dagger}$ Roberto Cingolani, ${ }^{\dagger}$ and \\ Liberato Manna* ${ }^{*}$
}

${ }^{\dagger}$ Istituto Italiano di Tecnologia, via Morego 30, 16163 Genova, Italy, ${ }^{\dagger}$ Kavli Institute of Nanoscience, Delft University of Technology, Lorentzweg 1, 2628 CJ Delft, The Netherlands, and ${ }^{\S}$ EMAT, University of Antwerp,

Groenenborgerlaan 171, 2020 Antwerp, Belgium

\begin{abstract}
The thermal evolution of a collection of heterogeneous CdSe-Au nanosystems (Au-decorated CdSe nanorods, networks, vertical assemblies) prepared by wet-chemical approaches was monitored in situ in the transmission electron microscope. In contrast to interfaces that are formed during kinetically controlled wet chemical synthesis, heating under vacuum conditions results in distinct and well-defined CdSe/Au interfaces, located at the CdSe polar surfaces. The high quality of these interfaces should make the heterostructures more suitable for use in nanoscale electronic devices.
\end{abstract}

KEYWORDS Nanorods, nanocrystals, self-assembly, epitaxy, orientation relationship, interface, hybrid nanocrystals, gold, cadmium selenide, annealing

$\mathrm{C}$ olloidal inorganic nanocrystals of semiconductors are promising materials in a variety of applications. In photocatalysis and photovoltaics, for example, the photoinduced generation of charge carriers in nanocrystals can lead to the oxidation/reduction of molecular species or to the generation of clean electrical energy, respectively. ${ }^{1-3}$ In both cases, the performance of the material depends strongly on its charge separation ability and on the construction of suitable nanocrystal-electrode interfaces. The growth of metallic domains directly on the surface of the semiconductor nanocrystals can help to improve both factors. ${ }^{4-6}$ Banin's group reported the growth of Au domains on one or both tips of CdSe nanorods, ${ }^{7,8}$ and other studies have extended this approach to CdS nanorods, ${ }^{9} \mathrm{CdSe}(\mathrm{core}) @ \mathrm{CdS}-$ (shell) nanorods, ${ }^{10}$ and PbSe nanocrystals. ${ }^{11}$

Various metal nanoparticles can now be nucleated at selected locations of semiconductor nanorods, for example, at the tip regions, ${ }^{8,12}$ or close to where the seed is buried in seeded-growth core@shell nanorods, ${ }^{10}$ or yet throughout the nanorod surface. ${ }^{9,13}$ These nanostructures, characterized by metallic-semiconductor interfaces, represent a unique model for studying effects such as mixing of electron states, carrier injection, and charge separation processes. ${ }^{14,15}$ For the most investigated cases of wurtzite semiconductor nanorods of CdSe or CdS carrying metal domains at their tips, various optical, ${ }^{7,9}$ electronic, ${ }^{4,15}$ transport, $^{6}$ computational ${ }^{16,17}$ and photocatalytic ${ }^{18,19}$ studies have been reported. Thus the nature of the metal-semiconductor nanointerface and its

\footnotetext{
* To whom correspondence should be addressed. E-mail: liberato.manna@iit.it.

"These authors contributed equally to this work.

Received for review: 04/27/2010

Published on Web: 07/16/2010
}

influence on the local electronic structure of the nanorod have become subjects of particular interest in the past decade. The presence of metal $(\mathrm{Au})$ domains on specific regions of rods/wires has been additionally exploited for assembling them onto substrates and in solution., ${ }^{7,20-22}$

A major drawback of the samples used in these studies is that there appears to be no preferential epitaxial relationship between the metal and the cadmium chalcogenide domain. ${ }^{7,9}$ This is most likely a consequence of the mild conditions under which the growth of the metal on top of the nanocrystals is usually carried out. The lack of preferential crystallographic alignment is a key limitation for various potential applications, for example in nanoelectronics, because each metal-semiconductor nanointerface would behave in a somehow unique and unpredictable way. ${ }^{15}$ Consequently, the need for preferential alignment between $\mathrm{Au}$ and wurtzite cadmium chalcogenide materials is of significant importance.

The poor quality of colloidal nanocrystals involving Au and cadmium chalcogenide nanorods is further highlighted by the evidence that there is seldom an atomically flat and extended interface between the two materials. Often, so little is the number of atomic bonds between the semiconductor and the gold region that these bonds could be readily broken upon exposure to strong coordinating ligands, which could cause the heterostructures to break apart. Another limitation of the Au growth under mild conditions is that complete selectivity toward growth on one specific region on the nanocrystal surface is actually hard to achieve: the nucleation of many small ( $1-3 \mathrm{~nm}$ diameter) Au domains on various regions along the surface of the rods often competes with the growth of $\mathrm{Au}$ on the basal polar facets, ${ }^{7-10,23}$ most likely 

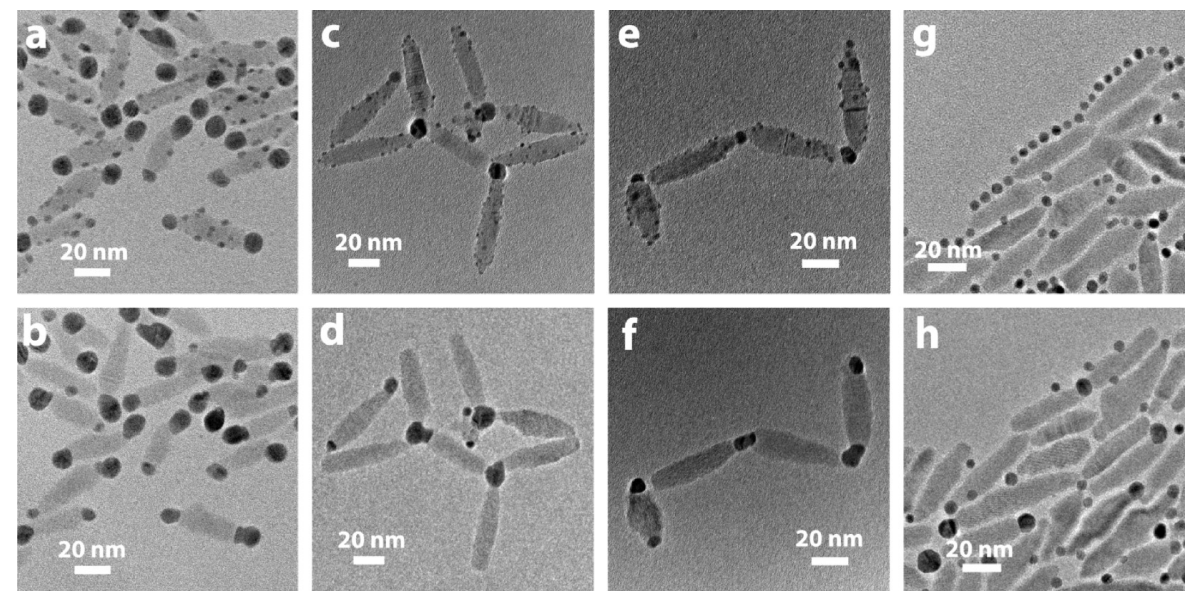

FIGURE 1. TEM images of various CdSe-Au nanorod heterostructures and of nanocrystal mixtures as-deposited (a,c,e,g) and after annealing at $200-250{ }^{\circ} \mathrm{C}(\mathrm{b}, \mathrm{d}, \mathrm{f}, \mathrm{h})$. The samples before annealing are as follows: (a) isolated, Au-decorated CdSe nanorods; (c,e) assemblies of nanorods welded to each other via Au domains; and (g) a mixture of isolated Au nanocrystals and CdSe nanorods. The samples after annealing are as follows: (b) in Au-tipped nanorods, after annealing, all small Au clusters along the sides of the rods had disappeared, feeding the Au domains at the rod tips; $(d, f)$ in network structures, the Au domains linking more than two CdSe nanorods where those that preferentially increased in volume, even at the expense of small Au clusters initially present on the rod tips at the far ends of the junctions; (h) upon annealing a mixture of isolated nanorods and Au nanocrystals, new Au crystals having an interface with CdSe were formed at the rod tips. ${ }^{30}$

because those regions have surface defects or are not efficiently passivated by surfactants.

Di Felice et al. predicted recently by ab initio calculations the possibility to obtain stable Au-CdX (hexagonal) interfaces with controlled atomic mixing, crystal orientation and plasmonic-excitonic interactions. ${ }^{16,17} \mathrm{~A}$ question arises therefore on the likelihood that, starting from wurtzite nanorods, heterostructures could be fabricated with a preferential orientation between the Au and the semiconductor lattices. Hence, an extended and flat interface between the two materials, and a strong selectivity for Au growth at nanorod basal facets could be expected in these cases. It is also known that annealing of interfaces, especially those involving $\mathrm{Au}$ as one component, can lead to substantial interface reconstruction due to the relatively high surface mobility of Au atoms. ${ }^{24,25}$

In the present work, we investigate the structural and morphological evolution of as-synthesized, defective CdSe nanorods decorated with gold domains, deposited on a substrate and subjected to thermal annealing. We show that such treatment indeed leads to heterostructures with better defined metal-semiconductor interfaces. The advantage of performing this process on a substrate, in the absence of surfactants, solvent molecules, and unreacted chemical precursors, is that many processes like nanocrystal growth, dissolution, or severe reshaping, are minimized. In the present work, this evolution could be additionally followed in situ, using an aberration-corrected transmission electron microscope (TEM; FEI Titan operating at $300 \mathrm{kV}$ ) and a lowdrift heating holder, so that structural and shape transformations in individual nanocrystals could be imaged in real time and with atomic resolution. ${ }^{26}$

Various CdSe-Au nanocrystal samples were prepared using wet-chemical approaches, starting from CdSe nano- rods, ${ }^{27}$ all following previously published recipes or modifications thereof. The samples studied were (i) nanorods with Au domains grown at one or both rod tips, ${ }^{7}$ (ii) nanorods homogeneously decorated with Au domains throughout their surface, (iii) assemblies of nanorods welded to each other at their tips through their Au domains, ${ }^{20}$ (iv) mixtures of isolated nanorods (laying flat on the substrate) and isolated spherical Au nanocrystals, ${ }^{28}$ and (v) bundles of hexagonally close-packed nanorods vertically standing on a substrate, where each nanorod carries several small Au nanocrystals all over its surface. ${ }^{29}$ The various samples were prepared for TEM by simply depositing one drop of solution of nanocrystals (or nanocrystal assemblies) onto the SiN membrane of a microheater device fabricated using MEMS technology (microelectronic mechanical systems). ${ }^{26}$ After complete solvent evaporation was observed, the chips were inserted in the vacuum of the TEM and their thermal evolution was monitored in situ, from room temperature up to $400{ }^{\circ} \mathrm{C}$. Accurate control of the temperature was achieved through feedback electronics and software, whereby every MEMS microheater was calibrated beforehand by determination of the resistancetemperature relationship. ${ }^{26}$

In "as-deposited" samples investigated with TEM at room temperature, many small Au clusters ( $1-3 \mathrm{~nm}$ in diameter) were attached to the lateral surface of the rods, in addition to the larger Au domains located at the rod tips (Figure $1 \mathrm{a}, \mathrm{c}, \mathrm{e})$. Heating the samples between 200 and $250{ }^{\circ} \mathrm{C}$ caused an enlargement of the Au domains at the tips. At the same time, the small gold clusters initially distributed throughout the CdSe lateral surface either decreased in size until they disappeared, or they migrated altogether to the tips (Figure $1 \mathrm{~b}, \mathrm{~d}, \mathrm{f})$. This could be followed in real time, that is, these transformations were seen occurring under the electron beam while the temperature of the chip was increased. 
Annealing of samples decorated with Au nanocrystals induced mainly intrarod ripening, that is, in each rod the increase in volume of the Au domains at the tips could be correlated with the disappearance/migration of the smaller Au clusters initially located on its sides. No enlargement of Au domains was seen instead at the expense of Au located on other nanorods, that is, no inter-rod ripening occurred.

In networks of nanorods (Figure 1c, d and e,f), in general, all Au domains at the rod tips, both those gluing nanorods together and those located at the far ends of the assemblies, showed the tendency to increase their volume (Figure 1e,f). However, the Au domains that most strongly increased in volume, even at the expense of those at the far ends of the assemblies, were those that glued together more than two nanorods (compare in Figure 1 panels c,d with panels e,f). This is most likely due to the reduced mobility of the latter $\mathrm{Au}$ domains and the possibility to maximize their total interface area with the CdSe rods at these sites. Even annealing of a mixture of isolated CdSe nanorods and spherical Au nanocrystals eventually led to the disappearance of most isolated Au nanocrystals from the substrate, and to the formation of large Au nanocrystals mainly at the tips of most nanorods (Figure $1 \mathrm{~g}, \mathrm{~h}$ ). Several ripening processes could be followed in real time, as shown in the Supporting Information Movie M1 for the case of isolated Au-decorated CdSe nanorods.

It was verified that all the observed structural transformations were due to thermal annealing and not to electron beam exposure. When examining other areas of the chip that had not been irradiated yet, the nanocrystals had undergone the same structural transformations as described above. The transformations could also be reproduced by means of ex-situ annealing in combination with SEM (scanning electron microscopy) examination. Here the same samples were dropcast onto silicon substrates, annealed exsitu under inert atmosphere at the same temperature as in the TEM experiments, and imaged in the SEM. A few examples of the outcome of these test experiments are reported in Figure 2 (see also Supporting Information for experimental details). The structural transformations reported here therefore follow a different pathway than those which are clearly triggered by photophysical processes and described in previous studies. ${ }^{23,24,31}$

The structural evolution of $\mathrm{CdSe}-\mathrm{Au}$ nanorod heterostructures in solution and at room temperature was studied by Banin et al. ${ }^{8}$ The authors found that nanorods decorated with multiple Au clusters on their surface evolved to nanorods carrying only one large Au crystal at one of their tips. The mechanism underlying that evolution was identified as an "intrarod" Ostwald electro-ripening driven by the sizedependence of the redox potential in metals. Namely, smaller Au clusters at the lateral surface of the rods were oxidized, releasing Au species in solution, which were again reduced at the bigger and now electron-rich Au domains at the tips of the rods. The same competing process then

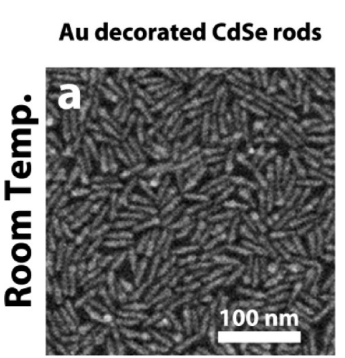

Au spherical nanocrystals + CdSe rods
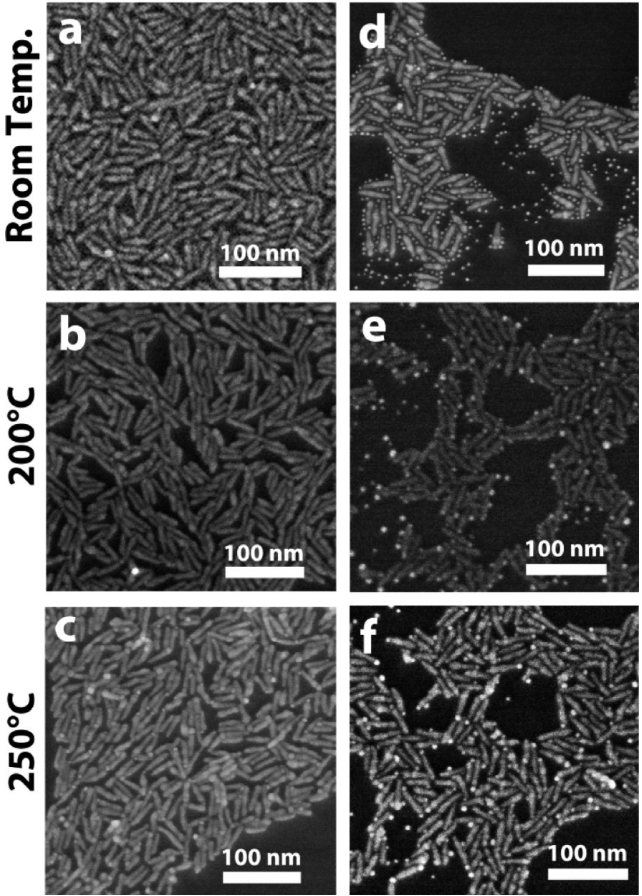

FIGURE 2. SEM images of two different CdSe-Au nanorod samples, deposited on a silicon substrate, before and after annealing at two different temperatures. For each sample, every micrograph refers to a different area on the same substrate. (a) As-deposited CdSe nanorods decorated with Au nanocrystals throughout their surface. The size of the Au nanocrystals here is so small that it is difficult to identify them under SEM. (b) After the sample was annealed at 200 ${ }^{\circ} \mathrm{C}$ for $10 \mathrm{~min}$, a few bright spots could be located on the tips of some rods, which is indicative of larger Au domains forming there. (c) After annealing at $250{ }^{\circ} \mathrm{C}$ for $10 \mathrm{~min}$, the number and size of these brighter spots increased, and they were all located at the tip regions of the rods. (d) A mixture of isolated Au spherical nanocrystals and CdSe nanorods, as-deposited on a silicon substrate. (e) In the same sample, after annealing at $200{ }^{\circ} \mathrm{C}$ for $10 \mathrm{~min}$, many nanorods seemed to have acquired one or more Au domains, mainly at their tips (but also a few were located on the side regions of the rods), and some "enlarged" isolated Au nanocrystals were also found throughout the substrate. (f) After further annealing at $250{ }^{\circ} \mathrm{C}$ for $10 \mathrm{~min}$, the number of isolated Au nanocrystals was reduced, while most Au nanocrystals were attached to the nanorods. A low affinity between the Au nanocrystals and the nanorods would have favored instead an independent $\mathrm{Au}$ ripening leaving the CdSe nanorods aside and leading to large isolated $\mathrm{Au}$ nanocrystals. ${ }^{32}$

occurred between the two Au domains at the opposite tips of the rods, so that eventually only the bigger domain survived and was actually enlarged in size. Analogous electroripening processes were observed by others for Ag nanoparticles deposited on conductive substrates and exposed to ionic solutions. ${ }^{33}$

In our experiments, all transformations took place instead in dried samples, at high temperature, and under vacuum/ inert atmosphere. Under these conditions, the formation of ionic Au species that could be transferred from different regions along the surface of a rod, or from rod to rod, should not occur, since there was no solution medium present capable to sustain such ionic diffusion process. Therefore, 

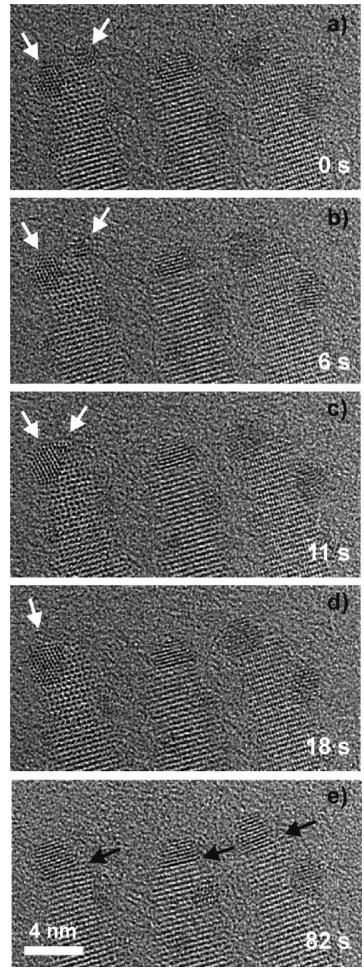

FIGURE 3. Stills of a movie (Supporting Information Movie M2) recorded during over $2 \mathrm{~min}$, during which a sample was heated from 170 to $190{ }^{\circ} \mathrm{C}$ at a constant rate. The time is indicated in each frame. White arrows in panels $(\mathrm{a}-\mathrm{d})$ point to two coalescing Au dots. The smallest Au dot ( $\sim 1 \mathrm{~nm}$ ) moved over the CdSe surface (and over the SiN support) until it fused with the larger Au dot at its left. Only in the central rod, the CdSe/Au interface at the tip was flat from the beginning. After heating for $82 \mathrm{~s}$, well-defined flat CdSe/Au interfaces were also formed at the tips of the other two rods (black arrows in panel e).

alternative mechanisms such as cluster diffusion ${ }^{34}$ and diffusion of atoms ${ }^{35-37}$ are most likely operative in the evolution of nanocrystals deposited on substrates and annealed under vacuum (see also Supporting Information for more details on both mechanisms). Cluster diffusion in our samples was supported by in situ TEM movies recorded at temperatures between 150 and $250{ }^{\circ} \mathrm{C}$ (see Figure 3 and Movie M2 of the Supporting Information); $1-3 \mathrm{~nm}$ size Au clusters close to the tip regions of nanorods were seen to migrate along the rod surface until they merged with the largest Au domain already located at the tips. Atomic diffusion appeared to be operative instead for smaller Au clusters and/or clusters located further away from the tip regions of isolated nanorods or from the Au junctions of nanorod networks. These clusters did not tend to migrate, but they rather shrunk in size until they disappeared, while at the same time the Au domains at the rod tips/junction regions became larger (see Movie M3 and M4 of the Supporting Information). It is plausible that in many cases the structural evolution of nanorods occurred via both cluster and atomic surface diffusion (see Scheme 1).

In all cases that we have analyzed, starting from Audecorated nanorods or from networks of nanorods welded
SCHEME 1. Sketches of the Ostwald Ripening Mechanism, Occurring via Atomic Diffusion, and of the Cluster Diffusion Mechanism $^{a}$

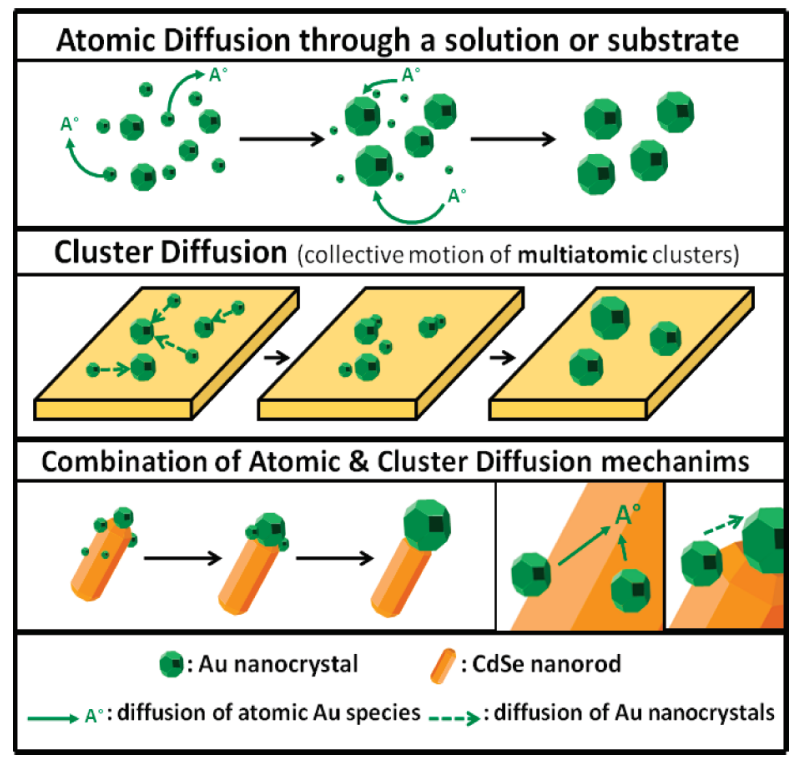

${ }^{a}$ The steady disappearance of Au nanocrystals from the lateral facets of CdSe nanorods during annealing can be ascribed to a combination of both mechanisms.

by gold domains, only intrarod ripening was observed, hence involving diffusion of Au clusters or atoms along the surface of the nanorods. When annealing mixtures of isolated Au particles and nanorods, the evolution of Au domain at the tips of some rods could reach sizes such that an Au domain at some point could bridge two or more rods that were previously separated (see Figure $1 \mathrm{~g}, \mathrm{~h}$ ). After this moment, inter-rod ripening processes could be also operative, but only via surface diffusion over the Au domain bridging the rods.

Apart from an increase in the size of the Au domains at the tips of the rods, their interface with the underlying CdSe lattice reconstructed considerably, that is, it became flatter and more extended than in the starting heterostructures (this can be seen already in Figure 3). The evolution of a single $\mathrm{CdSe} / \mathrm{Au}$ interface was also followed in real time at atomic resolution, while raising the temperature of the sample between 150 to $300{ }^{\circ} \mathrm{C}$ at a high heating rate $(2 \mathrm{~K} / \mathrm{s})$. The degree of interfacial reconstruction is shown in Figure $4 a-c$. No significant alloying between Au and CdSe occurred at any temperature, as at every stage of the heating the lattice parameters of both domains remained constant and close to the bulk values. The conditions at which the experiments were performed are clearly different from the dynamic conditions in solution under which these heterostructures are usually synthesized ${ }^{7,9}$ and are much closer to thermodynamic equilibrium.

All these results provide strong evidence of the high stability of the newly formed CdSe/Au interface. Indeed, an epitaxial relationship was found between the CdSe and the Au domains after annealing at $250{ }^{\circ} \mathrm{C}$, in contrast to the 

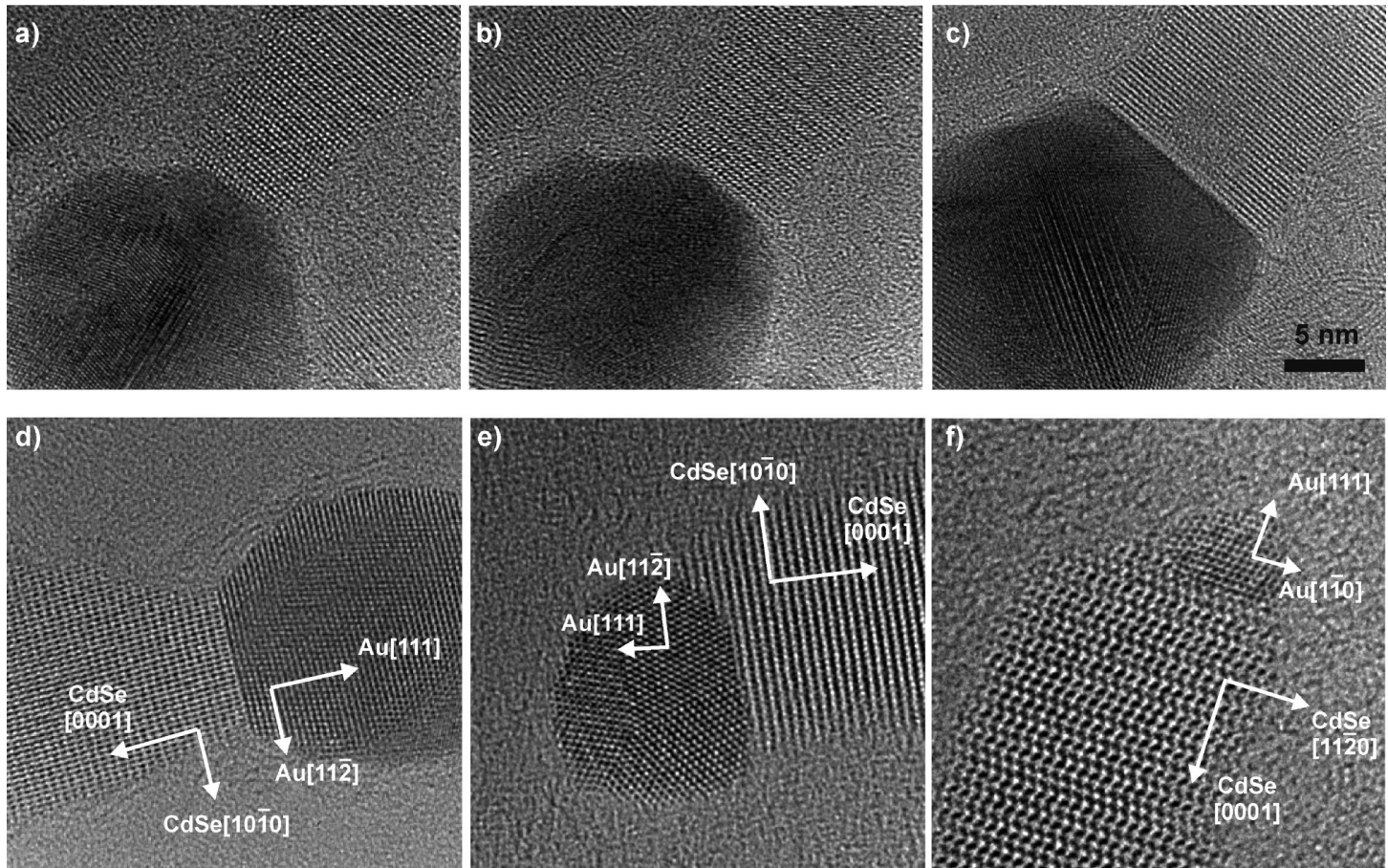

FIGURE 4. Enlargement and flattening of the CdSe/Au interface $(a-c)$. During heating at a high heating rate $(2 \mathrm{~K} / \mathrm{s})$, the width of the interface grew by a factor of 3 . Images are shown corresponding to temperatures of $150^{\circ} \mathrm{C}$ (a), $250^{\circ} \mathrm{C}$ (b), and $300^{\circ} \mathrm{C}$ (c), respectively. Such an interface reconstruction enables the two crystal lattices to accommodate to each other to maximize the number of covalent bonds between them. In the bottom row of the figure, various CdSe/Au interfaces with both the CdSe and the Au crystals in identifiable zone axes are shown ( $d-f)$. All configurations show the same epitaxial relationship, as indicated in the figure. (d) Large Au domain; (e) decahedral Au crystal, whereby one subcrystal (upper side of the interface) follows the preferential orientation while the second subcrystal (bottom side of the interface) has a misaligned interface. (f) Small Au domain.

random orientations of the as-synthesized nanocrystals. In all cases, the CdSe $\{0001\}$ atomic planes were aligned with the $A u\{111\}$ atomic planes, see for instance Figure $4 d-f$. On the basis of the analysis of several heterostructures, a full preferential orientation relationship was determined as:

\section{CdSe $\{0001\} / A u\{111\}$ and CdSe[1010]/Au[1 12]}

A direct comparison with bulk heteroepitaxial interfaces can be made by considering for example the highly crystalline thin films of CdSe grown by Stickney et al. on (111) oriented Au substrates via electrochemical atomic layer epitaxy. ${ }^{38}$ The CdSe films exhibited in that case a cubic zincblende structure with a (111) preferred orientation with respect to the substrate. ${ }^{39}$ Since the $\{0001\}$ facets of wurtzite CdSe and the $\{111\}$ facets of zinc-blende CdSe are equivalent in terms of symmetry, positions of atoms and of bonds, the epitaxial relationship found by us is practically the same as that reported for bulk interfaces. The epitaxy can be rationalized by considering that two surface unit cells of the CdSe $\{0001\}$ facets match with three surface unit cells of the Au\{111\} facets. Such periodicity leads to a commensurate epitaxial relationship with low mismatch values, which are equal to $3.2 \%$ in both directions along the interfacial plane (see also Supporting Information for more details)
The higher stability of this interface with respect to other possible interfaces can be easily understood if one considers that the $\{0001\}$ facets of $\mathrm{CdSe} e^{40,41}$ are the least stable ones. Therefore, their energy is minimized by the formation of the heteroepitaxy. ${ }^{42}$ The free CdSe $\{0001\}$ surface is polar, since along the [0001] direction the crystal consists of alternating positively charged atomic layers ( $\mathrm{Cd}$ atoms) and negatively charged atomic layers (Se atoms). Consequently, the crystal ends with an electric dipole at the surface, which has a high energetic cost. However, when an interface with $\mathrm{Au}$ is formed, the dipole is accommodated by the free electron gas of the metal. It is known from the theory of ceramic-metal interfaces ${ }^{43}$ that because of this charge transfer, the work of adhesion for polar/metal interfaces is higher than for nonpolar/metal interfaces, so that the interface energy is much reduced in the case of polar/metal interfaces. Therefore, apart from the structural commensurate fit, it is plausible that the electronic configuration at the interface promotes the formation of the CdSe $\{0001\} / A u$ interface. It was shown already computationally that charge transfer takes place in the related, polar-CdS $\{0001\} / A u$ system, although no comparison was made with nonpolar-CdS/Au systems. ${ }^{17}$ Some experimental evidence have confirmed this charge transfer in metal-semiconductor nanostructures. ${ }^{4,18,44}$ On the other hand, the $\mathrm{Au}\{111\}$ facet shows the lowest surface energy value of the crystal and is clearly the most 
stable one, as reflected in the tendency of thin-film growth to propagate in the [111] direction. ${ }^{45-47}$ The high mobility of Au atoms $s^{24,25}$ facilitates its reconstruction on the CdSe surface during the annealing process. Therefore, a larger and well-defined $\{111\}$ surface is developed.

Not to forget is the well-known catalytic activity of Au clusters, in particular that of the Au\{111\} surfaces. The interface involving this facet has been often involved in catalytic processes both at the nanoscale or at the molecular level: the growth of a second inorganic particle onto the $\mathrm{Au}$ cluster surface ${ }^{11,48-51}$ or several heterogeneous catalytic molecular processes ${ }^{52}$ are usually mediated by $\mathrm{Au}\{111\}$ facets. Consequently, in our case it can be reasonably hypothesized that the same facet helps to better accommodate the electric dipole of the polar CdSe facets. Zeng et al. recently reported on spherical CdSe-Au hybrid nanocrystals with a different epitaxial relationship. ${ }^{53}$ However, in that case, the ambient and surfactant-assisted conditions under which the heterostructures were formed make it difficult to assess it as the most stable configuration.

On the basis of what has been reported for other $\mathrm{Au}$ based hybrid nanocrystals ${ }^{48,49,51,54}$ (see also the Supporting Information for more details) and taking into consideration our previous observations, it seems that high temperatures are required to overcome the energy barrier that allows the interface between CdSe and Au to reach its thermodynamically most stable configuration, hence to achieve a clear epitaxial relationship between the two lattices. In principle, such a stable interface can be fabricated under conditions that differ from those involving annealing on a substrate, as long as sufficiently high temperatures can be reached. To this aim, we synthesized CdSe-Au heterostructures in solution, using an approach that should bring the system to conditions close to thermodynamic equilibrium. We mixed together isolated CdSe nanocrystals (either nanorods or spherical shaped nanoparticles) and Au nanocrystals in a high boiling noncoordinating solvent such as octadecene, in the presence of a small amount of oleylamine to guarantee their colloidal stability. After the solution was heated at 250 ${ }^{\circ} \mathrm{C}$ for $1 \mathrm{~h}$, Au-tipped CdSe "nanoworms" were formed, as shown in Figure 5.

These heterostructures are characterized by the same preferential orientation between CdSe and Au as found in the previous samples after annealing. Elemental mapping however indicated the presence of atomic $\mathrm{Au}$ in the hexagonal CdSe domain (see Supporting Information for the EDX analysis). Au atoms were found to occupy mainly Se positions of the wurtzite CdSe crystal lattice and their concentration increased when moving closer to the CdSe/ $\mathrm{Au}$ interface. This gradient of $\mathrm{Au}$ atoms induced also a significant and gradual decrease of wurtzite CdSe typical lattice parameters, as systematically observed by Fourier analysis of the HRTEM images, which could be the reason for the curved "wormlike" morphology of the CdSe domain. Most frequently, the curved CdSe rods were found to be
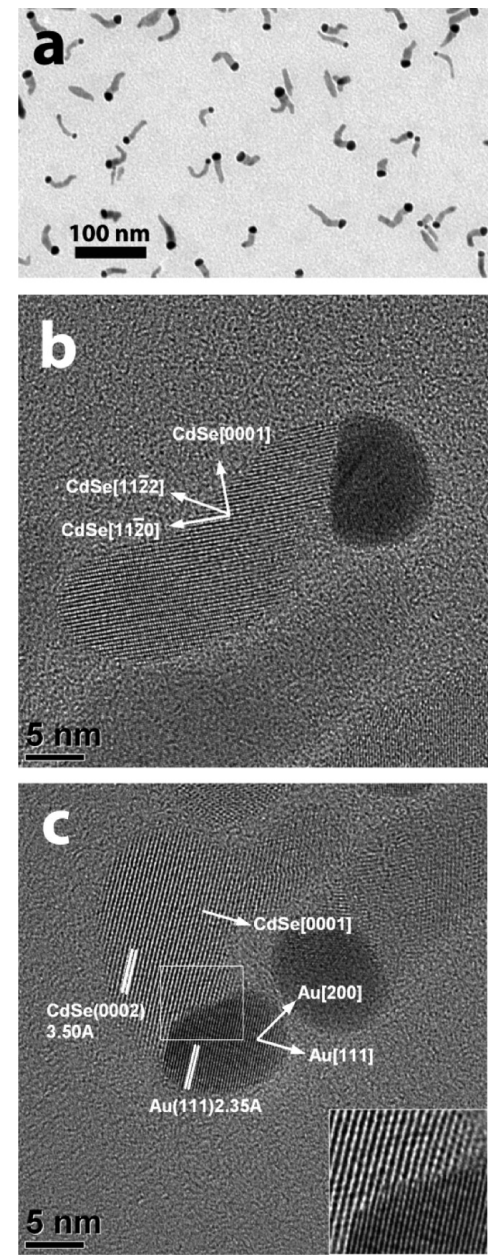

FIGURE 5. Low-resolution (a) and high-resolution (b,c) TEM images of epitaxially grown CdSe-Au nanocrystals synthesized at $250^{\circ} \mathrm{C}$ by wet chemical synthesis. In the high-resolution images, the monocrystalline character of the wormlike CdSe (b) and the rod preferential orientation with respect to the Au domain (c) are respectively shown. (Inset of panel c) Detailed image of the defects located at the CdSe/Au interface.

monocrystalline, as imaged in Figure 5b. However, similar to what has been observed by Korgel et al. in $\mathrm{Au}-\mathrm{Si}$ nanorods ${ }^{55}$ in some cases such a curved morphology can be also correlated to the presence of defects along the rod (see Figure S3 in Supporting Information). The mechanism by which such heterostructures are formed in solution is based on an initial "dismantling" of the starting CdSe nanocrystals, followed by their recrystallization on the Au surface (see Figure S4 of the Supporting Information). During this recrystallization process, Au atoms are inserted in the CdSe structure, probably due to the presence of Au atomic species in solution during a concomitant Oswald ripening process of the Au domains. It is unlikely that the growth of these CdSe "worms" proceeds via a solution-liquid-solid (SLS) growth mechanism mediated by the presence of Au nanocrystals in solution, similar to what was previously observed by Buhro et al. when growing bicatalyzed metal sulphide and selenide nanowires ${ }^{56,57}$ or by Korgel et al. when growing Au- 
catalyzed silicon nanorods. ${ }^{55}$ Indeed, the temperature at which these structures are formed is well below the CdSe/ $\mathrm{Au}$ eutectic temperature, which is required in the SLS growth. It is more plausible therefore that the growth proceeds through some sort of heterogeneous nucleation of CdSe on top of the Au nanocrystals (one could call this a "solution-solid-solid" growth mode, as opposed to the SLS mechanism). In any case, these results corroborate our previous assumptions on thermally achievable CdSe/Au stable interfaces.

One of the most interesting applications that could be derived from these thermally induced phenomena concerns the potential for in situ fabrication of metallic electrodes on top of preassembled nanocrystals. The assembly of optically active nanocrystals, especially of those showing a high degree of shape control, is a crucial aspect for the fabrication of electronic devices. In the last years, several groups have developed different methodologies for the micrometer-size organization of nanocrystals on substrates. ${ }^{27,29,58-60}$ However, after the assembly of the active units in the device, the formation of well-defined and high quality nanocrystalelectrode contacts poses a further challenge. Driven by the aim to find new approaches to build metal contacts on top of (or sandwiching) nanocrystal layers, we performed a simple experiment. We first assembled CdSe nanorods, which had been previously decorated with small Au clusters, in close packed arrays vertically standing on a substrate, using depletion attraction forces (see Figure 6a). ${ }^{29}$ These assemblies were then thermally annealed. As observed in all previously described examples, the thermal ripening of Au occurred along the vertically assembled rods, and larger Au domains were formed gradually on top of the rods during the annealing between 150 and $300{ }^{\circ} \mathrm{C}$, as seen in Figure $6 \mathrm{~b}-\mathrm{e}$ and Movie M6 of the Supporting Information. Many of these domains had coalesced together, due to the close proximity of nanorods in the assemblies. Hence, large Au nanocrystals ended up bridging several nanorod tips, both on the top and on the bottom side of the monolayers of vertically standing rods, as shown in Figure 6. These preliminary results represent a starting point for the in situ formation of metallic electrodes in nanostructured electronic devices, or alternatively for the improvement of the electric contact between the active material and preformed electrodes, by means of the temperature induced reconstruction of their interface.

In summary, we provide clear evidence of the temperature-dependent evolution of the interface in CdSe-Au heterostructures by means of in situ annealing high-resolution TEM measurements. Our work demonstrates into detail the effective ripening of Au nanocrystals that leads to a selective positioning of $\mathrm{Au}$ domains at the tips of rod-shaped CdSe nanocrystals. The real-time TEM measurements, performed while heating the sample, allowed identifying the migrationbased ripening mechanism by which some Au nanocrystals coalesce to enlarge the Au domains at the tips. We further
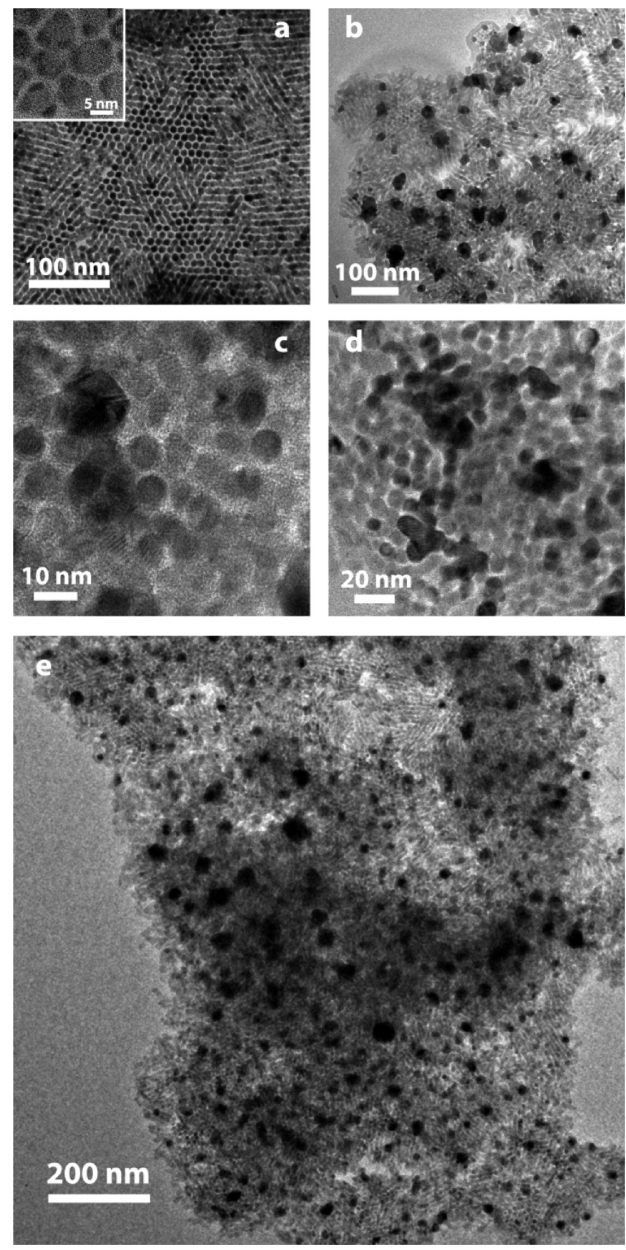

FIGURE 6. Monolayers of close-packed CdSe nanorods, vertically standing on the silicon nitride membrane, and decorated with small $\mathrm{Au}$ clusters before (a) and after the annealing treatment at $250^{\circ} \mathrm{C}$ (b). In the inset of panel a, the lateral $\mathrm{Au}$ decoration of the as-prepared vertically standing $\mathrm{CdSe}$ rods is shown. Panels c, d show details of the annealed samples, where $\mathrm{Au}$ islands formed are seen to connect several CdSe nanorods. An expanded area of the annealed assemblies is shown in panel e, where $\mathrm{Au}$ islands are seen to bridge several nanorod tips.

demonstrate that the ripening at high temperatures is accompanied by a global interface reconstruction between the two domains at the tips. This phenomenon enables the formation of larger and better defined interfaces, whereby the number of covalent bonds between the two materials is maximized. The main driving force of these thermal phenomena is the formation of a preferential orientation between the Au and the CdSe crystallographic lattices, which we induced by bringing the configurations toward thermodynamic equilibrium conditions.

Metallic-semiconductor interfaces are crucial in electronic and photovoltaic devices. Hence, a method for fabricating well-defined interfaces between metal and semiconductor nanocrystals should foster systematic studies of their electronic structure and conductive characteristics. In this direction, we have also indicated the possibility to form $\mathrm{Au}$ conductive contacts on the top/bottom surfaces of preas- 
sembled and vertically standing CdSe nanorods, simply by thermal annealing. Finally, our approach could represent a general procedure for the epitaxial growth of different metal domains (in addition to $\mathrm{Au}$ ) on nanocrystalline materials other than II-VI semiconductors. As an example, synthetic procedures for the wet-chemical preparation of nanosized oxide-metal particles are still uncommon and difficult to generalize ${ }^{61}$ and these difficulties might be overcome by thermal annealing of the appropriate building blocks, which could lead to stable metal-oxide nanointerfaces.

Acknowledgment. The authors acknowledge financial support from the European Union, through the FP7 program (ERC Starting Grant NANO-ARCH, Contract Number 240111) and the UF6 program (I3, ref. 026019 ESTEEM).

Supporting Information Available. Thermally induced ripening of $\mathrm{Au}$ nanocrystals decorating CdSe nanorods; details on the ex-situ annealing experiments of different types of nanocrystals on Si substrates; SEM images of Au nanocrystals deposited on Si substrates before and after exsitu annealing at $250{ }^{\circ} \mathrm{C}$; discussion on the Au ripening mechanisms and movies supporting the statements made in the main text; details on the calculations of CdSe/Au lattice mismatch; description and discussion on the high temperature induced CdSe sublimation; discussion on the experimental conditions for the synthesis of other epitaxial Aubased hybrid nanoparticles; elemental mapping analysis of the solution-prepared CdSe-Au "wormlike" heterostructures; high resolution TEM image showing the presence of defects in the polycrystalline wormlike wurtzite CdSe domain of CdSe-Au nanocrystals synthesized by wet chemical approaches; temporal evolution of the photoluminescence spectrum during the growth of CdSe-Au wormlike heterostructures; thermally induced ripening process of $\mathrm{Au}$ in assemblies of Au-decorated CdSe nanorods standing vertically on silicon nitride substrates. This material is available free of charge via the Internet at http://pubs.acs.org.

\section{REFERENCES AND NOTES}

(1) Talapin, D. V.; Lee, J. S.; Kovalenko, M. V.; Shevchenko, E. V. Chem. Rev. 2010, 110, 389-458.

(2) Li, J. H.; Zhang, J. Z. Coord. Chem. Rev. 2009, 253, 3015-3041.

(3) Hernandez-Alonso, M. D.; Fresno, F.; Suarez, S.; Coronado, J. M. Energy Environ. Sci. 2009, 2, 1231-1257.

(4) Costi, R.; Cohen, G.; Salant, A.; Rabani, E.; Banin, U. Nano Lett. 2009, 9, 2031-2039

(5) Lee, H.; Yang, H.; Holloway, P. H. Physica B 2009, 404, $4364-$ 4369.

(6) Sheldon, M. T.; Trudeau, P. E.; Mokari, T.; Wang, L. W.; Alivisatos, A. P. Nano Lett. 2009, 9, 3676-3682.

(7) Mokari, T.; Rothenberg, E.; Popov, I.; Costi, R.; Banin, U. Science 2004, 304, 1787-1790.

(8) Mokari, T.; Sztrum, C. G.; Salant, A.; Rabani, E.; Banin, U. Nat. Mater. 2005, 4, 855-863.

(9) Saunders, A. E.; Popov, I.; Banin, U. J. Phys. Chem. B 2006, 110 , 25421-25429.

(10) Menagen, G.; Mocatta, D.; Salant, A.; Popov, I.; Dorfs, D.; Banin, U. Chem. Mater. 2008, 20, 6900-6902.

(11) Shi, W. L.; Zeng, H.; Sahoo, Y.; Ohulchanskyy, T. Y.; Ding, Y.; Wang, Z. L.; Swihart, M.; Prasad, P. N. Nano Lett. 2006, 6, 875881
(12) Chakrabortty, S.; Yang, Jie A.; Tan, Yee M.; Mishra, N.; Chan, Y. Angew. Chem., Int. Ed. 2010, 49, 2888-2892.

(13) Plante, I. J. L.; Habas, S. E.; Yuhas, B. D.; Gargas, D. J.; Mokari, T. Chem. Mater. 2009, 21, 3662-3667.

(14) Gooding, A. K.; Gómez, D. E.; Mulvaney, P. ACS Nano 2008, 2, 669-676.

(15) Steiner, D.; Mokari, T.; Banin, U.; Millo, O. Phys. Rev. Lett. 2005, 95, No. 056805.

(16) de Paiva, R.; Di Felice, R. ACS Nano 2008, 2, 2225-2236.

(17) de Paiva, R.; Di Felice, R. J. Phys. Chem. C 2010, 114, 3998-4007.

(18) Costi, R.; Saunders, A. E.; Elmalem, E.; Salant, A.; Banin, U. Nano Lett. 2008, 8, 637-641

(19) Elmalem, E.; Saunders, A. E.; Costi, R.; Salant, A.; Banin, U. Adv. Mater. 2008, 20, 4312-4317

(20) Figuerola, A.; Franchini, I. R.; Fiore, A.; Mastria, R.; Falqui, A.; Bertoni, G.; Bals, S.; Van Tendeloo, G.; Kudera, S.; Cingolani, R.; Manna, L. Adv. Mater. 2009, 21, 550-554.

(21) Salant, A.; Amitay-Sadovsky, E.; Banin, U.J. Am. Chem. Soc. 2006, 128, 10006-10007.

(22) Ito, D.; Jespersen, M. L.; Hutchison, J. E. ACS Nano 2008, 2, 2001 2006.

(23) Menagen, G.; Macdonald, J. E.; Shemesh, Y.; Popov, I.; Banin, U. J. Am. Chem. Soc. 2009, 131, 17406-17411.

(24) Chen, Y.; Palmer, R. E.; Wilcoxon, J. P. Langmuir 2006, 22, 2851 2855.

(25) Baletto, F.; Mottet, C.; Ferrando, R. Surf. Sci. 2000, 446, 31-45

(26) van Huis, M. A.; Young, N. P.; Pandraud, G.; Creemer, J. F.; Vanmaekelbergh, D.; Kirkland, A. I.; Zandbergen, H. W. Adv. Mater. 2009, 21, 4992-4995

(27) Carbone, L.; et al. Nano Lett. 2007, 7, 2942-2950.

(28) Prasad, B. L. V.; Stoeva, S. I.; Sorensen, C. M.; Klabunde, K. J. Langmuir 2002, 18, 7515-7520.

(29) Baranov, D.; Fiore, A.; van Huis, M.; Giannini, C.; Falqui, A.; Lafont, U.; Zandbergen, H.; Zanella, M.; Cingolani, R.; Manna, L. Nano Lett. 2010, 10, 743-749.

(30) Most ripening processes could be followed real-time under exposure of the electron beam in the TEM, whereby the configurations formed in the examined areas were the same as configurations present elsewhere on the microheater chip (because the electron beam is small, and the membranes far apart, "fresh" areas could be checked frequently). Only in the case of the separated Au dots and CdSe rods (Fig. 1g,h), the electron beam irradiation apparently hampered the formation (nucleation) of new Au tips. So although this experiment is well reproducible both in the TEM and the SEM, no before/after images of the same area could be obtained. Possible explanations could be the irradiationinduced carburization of ligands that hampers changes (this seems unlikely because no such problems were encountered with the other nanostructures) or possibly the electron beam generates charging effects (electron-hole pairs in the cdSe rods or charge pile-up at the Au dots) which hampers the formation of new Au tips at the polar-CdSe/Au interface.

(31) Carbone, L.; Jakab, A.; Khalavka, Y.; Sönnichsen, C. Nano Lett. 2009, 9, 3710-3714.

(32) A control experiment, in which a substrate with only Au spherical nanocrystals was heated, is shown in Figure S1 of the Supporting Information.

(33) Redmond, P. L.; Hallock, A. J.; Brus, L. E. Nano Lett. 2005, 5, 131 135

(34) Smoluchowsky, M. v. Phys. Z. 1916, 17, 585

(35) Ostwald, W. Z. Z. Phys. Chem. 1901, 37, 385.

(36) Lifshitz, I. M.; Slyozov, V. V. J. Phys. Chem. Solids 1961, 19, 3550 .

(37) Wagner, C. Z. Elektrochem. 1961, 65, 581

(38) Colletti, L. P.; Flowers, B. H.; Stickney, J. L. J. Electrochem. Soc. $1998,145,1442-1449$

(39) Mathe, M. K.; Cox, S. M.; Flowers, B. H.; Vaidyanathan, R.; Pham, L.; Srisook, N.; Happek, U.; Stickney, J. L. J. Cryst. Growth 2004 , $271,55-64$.

(40) Manna, L.; Wang, L. W.; Cingolani, R.; Alivisatos, A. P. J. Phys. Chem. B 2005, 109, 6183-6192.

(41) Rempel, J. Y.; Trout, B. L.; Bawendi, M. G.; Jensen, K. F. J. Phys. Chem. B 2005, 109, 19320-19328. 
NANO

(42) See also the Supporting Movie M5 of the Supporting Information for further proofs on the stability of the CdSe/Au interface during the temperature-induced sublimation of the CdSe domain.

(43) Benedek, R.; Minkoff, M.; Yang, L. H. Phys. Rev. B 1996, 54, 7697 7700 .

(44) Subramanian, V.; Wolf, E. E.; Kamat, P. V.J. Am. Chem. Soc. 2004, $126,4943-4950$

(45) Gruznev, D. V.; Olyanich, D. A.; Chubenko, D. N.; Tsukanov, D. A.; Borisenko, E. A.; Bondarenko, L. V.; Ivanchenko, M. V.; Zotov, A. V.; Saranin, A. A. Surf. Sci. 2009, 603, 3400-3403.

(46) Devenyi, G. A.; Li, J. F.; Hughes, R. A.; Shi, A. C.; Mascher, P.; Preston, J. S. Nano Lett. 2009, 9, 4258-4263.

(47) Gonzalez-Gonzalez, A.; Sacedon, J. L.; Polop, C.; Rodriguez-Canas, E.; Aznarez, J. A.; Vasco, E. J. Vac. Sci. Technol., A 2009, 27, 10121016.

(48) Wetz, T.; Soulantica, K.; Talqui, A.; Respaud, M.; Snoeck, E.; Chaudret, B. Angew. Chem., Int. Ed. 2007, 46, 7079-7081.

(49) Zhang, H. T.; Ding, J.; Chow, G. M.; Dong, Z. L. Langmuir 2008, 24, 13197-13202.

(50) Yu, H.; Chen, M.; Rice, P. M.; Wang, S. X.; White, R. L.; Sun, S. H. Nano Lett. 2005, 5, 379-382.
(51) Pellegrino, T.; Fiore, A.; Carlino, E.; Giannini, C.; Cozzoli, P. D.; Ciccarella, G.; Respaud, M.; Palmirotta, L.; Cingolani, R.; Manna, L. J. Am. Chem. Soc. 2006, 128, 6690-6698.

(52) Gong, J. L.; Mullins, C. B. Acc. Chem. Res. 2009, 42, 1063-1073.

(53) Zeng, J.; Huang, J.; Liu, C.; Wu, C. H.; Lin, Y.; Wang, X.; Zhang, S.; Hou, J.; Xia, Y. Adv. Mater. 2010, 22, 1936-1940.

(54) Wei, Y.; Klajn, R.; Pinchuk, A. O.; Grzybowski, B. A. Small 2008, 4, 1635-1639.

(55) Heitsch, A. T.; Hessel, C. M.; Akhavan, V. A.; Korgel, B. A. Nano Lett. 2009, 9, 3042-3047.

(56) Yu, H.; Li, J. B.; Loomis, R. A.; Gibbons, P. C.; Wang, L. W.; Buhro, W. E. J. Am. Chem. Soc. 2003, 125, 16168-16169.

(57) Sun, J. W.; Buhro, W. E. Angew. Chem., Int. Ed. 2008, 47, 32153218.

(58) Talapin, D. V.; Shevchenko, E. V.; Murray, C. B.; Kornowski, A.; Förster, S.; Weller, H.J. Am. Chem. Soc. 2004, 126, 12984-12988.

(59) Ryan, K. M.; Mastroianni, A.; Stancil, K. A.; Liu, H.; Alivisatos, A. P. Nano Lett. 2006, 6, 1479-1482.

(60) Baker, J. L.; Widmer-Cooper, A.; Toney, M. F.; Geissler, P. L.; Alivisatos, A. P. Nano Lett. 2010, 10, 195-201.

(61) Casavola, M.; Buonsanti, R.; Caputo, G.; Cozzoli, P. D. Eur. J. Inorg. Chem. 2008, 2008, 837-854.

() 2010 American Chemical Society

3036

DOI: 10.1021/nl101482q | Nano Lett. 2010, 10, 3028--3036 